\title{
Possibilities without possible worlds/histories
}

\author{
Tomasz Placek *
}

October 16, 2009

\begin{abstract}
The paper puts forward a theory of historical modalities that is framed in terms of possible continuations rather than possible worlds or histories. The proposal is tested as a semantical theory for a language with historical modalities, tenses, and indexicals.
\end{abstract}

\section{Motivations}

Possible histories/worlds are philosophically demanding. They are posited to analyze either our modal discourse or indeterminism, or both. To qualify for this task they must be in some sense real, but in which sense exactly? Theoreticians of possible worlds/histories face a further problem of individuals: does an individual have counterparts in other possible worlds/histories, or does an individual have parts contained in those worlds/histories? Still further, if our world is indeterministic, the notion of 'actual history' does not seem to be legitimate. A related complaint is that we do not have epistemic access to histories, as these are differentiated by the minutest details, which might be located in a remote future. In contrast, possible continuations of events, or of stages of objects, appear to be innocuous as they are local and (can be thought of as arbitrarily) small. And our talk of possible continuations seems to be a natural translation of claims like: "I can sit but I can stand up as well". That is translated as: "There are two possible continuations of my present stage, in one I sit and in the other I stand".

*The paper owes very much to Nuel Belnap's hints, corrections, suggestions and comments. It was written during my visit to the Department of Philosophy at the University of Pittsburgh in February 2009. The visit was made possible by the Jagiellonian University WRBW grant and the MNiSW grant nr. 3165/32. For comments and useful suggestions I am also very grateful to Jacek Wawer and the audience of the Trends in Logic conference, Brussels, December 2008. 
There is also a theoretical concern motivating a search for a notion alternative to that of history. In the branching space-times of Belnap (1992) (henceforth BST 1992), two events do not belong to one history, if they do not have a common upper bound, where the ordering is defined in terms of light cones. In general relativity, even simple solutions (like Schwarzschild's solution) contravene this postulate as they allow for two events to be located in one space-time with no event above both of them. As a result, from the BST's (1992) perspective, a general relativistic (individual) space-time of this kind is viewed as a collection of possible histories. Finally, small and local objects like continuations fit the spirit of branching theories better than structures as large as histories. I take these motivations as sufficiently serious to attempt a refurbishment of BST theories. ${ }^{1}$ The paper aims at two goals: to construct a theory of possible branching continuations (BCont) and to show that it can serve as a semantics for a language with indexicals, historical modalities, and tenses. The further task of investigating whether the proposed theory can accommodate insights of general relativity is left for some later project.

\section{Mini-histories rather than histories}

In this section we assume rudiments of any branching theory: non-empty set $W$ partially ordered by $\leqslant$, with the usual interpretation. That is, $W$ is the set of possible point events, and $e \leqslant e^{\prime}$ means that $e^{\prime}$ lies in a possible future of $e$. Possible continuations of events should generalize elementary possibilities open at events of BST 1992. ${ }^{2}$ In an intended model of BST 1992 histories are copies of Minkowski space-time. Two histories splitting at a single choice event $e$ have separate future light-cones of $e$. The events that are space-like related to $e$ or are in the past light-cone of $e$ belong to the overlap of the two histories - see Figure (1). Notably, with the exception of $e$ itself, events

\footnotetext{
${ }^{1}$ By BST theories I mean, in the first place, N. Belnap's (1992) theory, but also some of its close kin, like Kowalski and Placek (1999) or Placek (2002).

${ }^{2}$ In BST 1992 an elementary possibility open at event $e$ is some particular subset $P$ of the set $H_{(e)}$ of histories passing through $e$; since possible continuations of $e$ are naturally thought as occurring above $e$, it is better to identify continuations with sets of particularly located events than to identify them with sets of histories. In this vein, a continuation $C$ corresponding to elementary possibility $P$ open at $e$ can be defined as $C=\{x \in \bigcup P \mid e<x\}$. Somewhat simpler, in Kowalski and Placek's (1999) framework continuations of $e$ can be identified with the so-called atomic outcomes of $e$. In both frameworks, however, continuations are history-dependent, and hence will not do for the present purpose.
} 

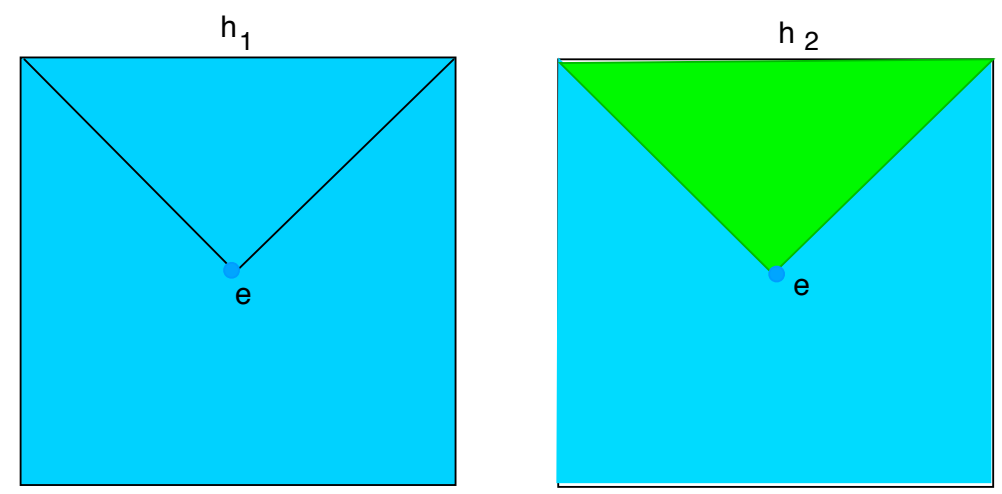

Figure 1: A BST 92 model with two histories $h_{1}$ and $h_{2}$, splitting at a single point $e$. The green area indicates where $h_{2}$ diverges from $h_{1}$. Note that no events on the future light-cone of $e$ belong to the overlap but $e$ is in the overlap.

on the future light-cone of $e$ do not belong to the overlap. This consequence of axioms of BST 92 permits a fruitful distinction between chanciness and indeterminism without choice. That is, an interval (i.e., a dense chain) joining an event in the overlap and some event above a choice event has different topological features, depending on whether or not it contains the choice event in question. In generalizing BST 92 we aim to retain this feature, and hence the above distinction.

A glimpse at a BST 92 model might suggest that $x$ and $y$ belong to a continuation of $e$ provided there is a V-like link above $e$, joining $x$ and $y$. In symbols, $\exists z \in W: e<z \wedge z \leqslant x \wedge z \leqslant y$. Yet, some events lying on the same future light-cone of $e$ cannot be joined by a V-link above $e$. We thus need to generalize V-links to (what we call): snake-links.

Definition 1 (snake-link)

$\left\langle e_{1}, e_{2}, \ldots, e_{n}\right\rangle \subseteq W(1 \leqslant n)$ is a snake-link iff

$$
\forall i: 0<i<n \rightarrow\left(e_{i} \leqslant e_{i+1} \vee e_{i+1} \leqslant e_{i}\right) .
$$

A snake-link is above (below) $e \in W$ if every element of it is strictly above (below) e.

Note that a single $x$ above $e$ as well as a chain above $e$ constitute snake-links above $e$.

\section{Definition 2 (snake-linked)}

For $x, y \in W, x$ and $y$ are snake-linked above $e, x \approx_{e} y$, iff there is a snakelink $\left\langle e_{1}, e_{2}, \ldots, e_{n}\right\rangle$ above $e$ such that $x=e_{1}$ and $y=e_{n}$. 
Analogously one may define the relations of being snake-linked below $e$, being snake-linked not-above $e$, being snake-linked not-below $e$, being snakelinked in a region, etc. For the record, we put down the definition of being snake-linked in a subset of $W$ :

\section{Definition 3 (snake-linked in a subset of $W$ )}

Let $W^{\prime} \subset W$ and $x, y \in W^{\prime} . x$ and $y$ are snake-linked in $W^{\prime}$ iff there is a snake-link $\left\langle e_{1}, e_{2}, \ldots, e_{n}\right\rangle$ such that $x=e_{1}$ and $y=e_{n}$ and $e_{i} \in W^{\prime}$ for every $0<i \leqslant n$.

Clearly, being snake-linked above a point event is a special case of being snaked-linked in a subset, yet for expository reasons, we present both the definitions.

Observe now that for any $x, y, z>e$, the following is true about being snake-linked above $e$ :

1. $x \approx_{e} x$

2. if $x \approx_{e} y$, then $y \approx_{e} x$;

3. if $x \approx_{e} y$ and $y \approx_{e} z$, then $x \approx_{e} z$.

Perhaps the third property requires an argument. Thus, let $x \approx_{e} y$ and $y \approx_{e} z$ be true due to the respective snake-links above $e,\left\langle e_{1}, \ldots, e_{n}\right\rangle$ and $\left\langle f_{1} \ldots, f_{m}\right\rangle$. Since $e_{n}=f_{1}=y$, the sequence $\left\langle g_{1}, \ldots, g_{m+n}\right\rangle$, such that $g_{k}=e_{k}$ if $k \leqslant n$ and $g_{k}=f_{k-n}$ if $n<k \leqslant m+n$, is a snake-link above $e$. Also, $g_{1}=x$ and $g_{n+m}=z$. Hence $x \approx_{e} z$.

The above properties mean that $\approx_{e}$ is an equivalence relation on set $W_{e}=\left\{e^{\prime} \in W \mid e<e^{\prime}\right\}$. Accordingly, $\approx_{e}$ induces a partition of set $W_{e}$. We will write this partition as $\Pi_{e}$ and call it the set of possible continuations of $e$. By $\Pi_{e}\langle x\rangle(e<x)$ we will mean the (unique) continuation of $e$ to which $x$ belongs. Events that have more than one possible continuation are called: choice events.

\section{Definition 4 (set $C E$ of choice events)}

For $e \in W, e \in C E$ iff $\operatorname{card}\left(\Pi_{e}\right)>1$.

There is no limitation on the number of possible continuations of events.

Consider now two pairs $\left\langle a, H_{a}\right\rangle$ and $\left\langle b, H_{b}\right\rangle$, where $a, b \in W$ and $H_{a} \in \Pi_{a}$, $H_{b} \in \Pi_{b}$. There is a sense in which the two pairs can be consistent. This occurs if $H_{a} \cap H_{b} \neq \emptyset$. To generalize the notion of consistency to arbitrarily large sets of pairs of this sort, we first recall the notion of pointer function.

Definition 5 (set $\mathcal{P}$ of pointer functions)

$f: W \rightarrow \bigcup_{e \in W} \Pi_{e}$ is a pointer function $(f \in \mathcal{P})$ iff $\forall_{e \in W} f(e) \in \Pi_{e}$. 
We will now say that $\langle A, f\rangle$, where $A \subset W$ and $f \in \mathcal{P}$ is consistent iff $\bigcap_{e \in A} f(e) \neq \emptyset$. A consistent pair of this sort will be called mini-history, or m-history for short:

\section{Definition 6 (m-histories)}

Pair $\langle A, f\rangle$ is an m-history iff $f$ is a pointer function with the domain restricted to $A \subset W$ and $\bigcap_{e \in A} f(e) \neq \emptyset$.

Note that in some cases a pointer function is redundant in specifying a mini-history, that is, to this end a subset of $W$ is enough. This occurs if $A \subset W$ is upper bounded and no maximal elements of $A$ are choice events. In this case there is a unique pointer function $f_{\mid A}$ with the domain restricted to $A$ that yields a mini-history $\langle A, f\rangle$ and which is defined as $f_{\mid A}(e):=\Pi_{e}\langle x\rangle$ for every $e \in A$, where $x$ is an upper bound of $A$. To see this, assume to the contrary that for some $e^{\prime} \in A: f\left(e^{\prime}\right) \neq \Pi_{e^{\prime}}\langle x\rangle$. Since $\Pi_{e^{\prime}}\langle x\rangle$ is a possible continuation of $e^{\prime}, e^{\prime}$ has at least two possible continuations, i.e., it is a choice event. Hence it cannot be a maximal element of $A$. There is thus $e^{\prime \prime} \in A$ such that $e^{\prime}<e^{\prime \prime}$. For $\langle A, f\rangle$ to be consistent, there must be $y$ such that $y \in f\left(e^{\prime}\right)$ and $y \in f\left(e^{\prime \prime}\right)$. Since $y \notin \Pi_{e^{\prime}}\langle x\rangle, y$ and $x$ are not snake-linked above $e^{\prime}$. However, since $y \in f\left(e^{\prime \prime}\right), e^{\prime \prime}<y$ and $e^{\prime \prime} \leqslant x$, and $e^{\prime}<e^{\prime \prime}$, so $x$ and $y$ are snake-lined above $e^{\prime}$. Contradiction.

Do not be beguiled into thinking that m-history is an analog of BT/BST history. It might be that $\langle\{a, b\}, f\rangle$ is not an m-history for the simple reason that there is nothing above both $a$ and $b$, and not because $a$ and $b$ belong to different continuations of some choice event.

Histories are defined in BT/BST as maximal upward directed subsets of the base set $W$. Could these be identified with maximal (in some sense) mhistories? But do the latter exist? To tackle these queries, let us introduce an ordering of m-histories:

\section{Definition 7 (ordering of m-histories)}

For m-histories $\langle A, f\rangle$ and $\langle B, g\rangle,\langle A, f\rangle \preccurlyeq\langle B, g\rangle$ iff $A \subseteq B$ and $g_{\mid A}=f$.

Clearly,

1. $\langle A, f\rangle \preccurlyeq\langle A, f\rangle$;

2. if $\langle A, f\rangle \preccurlyeq\langle B, g\rangle$ and $\langle A, f\rangle \succcurlyeq\langle B, g\rangle$, then $A=B$ and $f_{\mid A}=g_{\mid A}$, and hence $\langle A, f\rangle=\langle B, g\rangle$;

3. if $\langle A, f\rangle \preccurlyeq\langle B, g\rangle$ and $\langle B, g\rangle \preccurlyeq\langle C, d\rangle$, then $\langle A, f\rangle \preccurlyeq\langle C, d\rangle$.

To argue for the third clause, by the premises we have $A \subseteq B \subseteq C$. The right conjunct in the antecedent further entails $d_{\mid B}=g$, from which $d_{\mid A}=g_{\mid A}$ 
follows, given the inclusions above. From the left conjunct we have $g_{\mid A}=f$. These results together imply $d_{\mid A}=f$, as required.

It follows that $\preccurlyeq$ defined as above is a partial ordering on the set of $\mathrm{m}$ histories; further, the set is non-empty as long as the base set $W$ contains at least two comparable events. This condition, however, follows from the postulate that there are no maximal elements in $W$ - see the next section.

Having introduced the ordering, we state our claims. (1) In a non-empty partially ordered set $\langle W, \leqslant\rangle$, every chain can be extended to a maximal chain in $W$, and every upward directed subset of $W$ can be extended to a maximal upward directed subset of $W$. The first claim follows from the axiom of choice, the second is proved via the Zorn-Kuratowski lemma-for the proof, see Belnap (1992). Accordingly, since a model of the theory of branching continuations (BCont) is a non-empty partially ordered set subject to some postulates, it has maximal chains and maximal upward directed subsets of the base set, i.e., BT/BST histories. (2) Since we postulate that a base set $W$ has no maximal elements, every model of the theory of branching continuations has m-histories as well. (3) In some models there will be no maximal m-histories, and hence the notions of maximal m-history and of maximal upward directed subset of $W$ (or maximal chain in $W$ ) diverge.

Given the GR solutions for black holes, we recommend working with mhistories, and not ascribing any ontological significance to maximal upward directed subsets of base set $W$. This decline of the role of maximal upward directed subsets is somewhat reminiscent of how the status of maximal chains in a base set changes from BT to BST. In BT it is interpreted as a history, and in BST it has no ontological significance, as histories are identified with maximal upward directed subsets of $W$.

It remains to substantiate claim (3). To this end consider the real line $\Re$, naturally ordered. It is a model of BT, with a single BT history, and a model of BCont (check the axioms below!) with no choice point. Consequently, it permits only a single pointer function $f \in \mathcal{P}$ defined as follows: for $x$ in $\Re$ $f(x)=\{y \in \Re \mid x<y\}$. Consider now a chain of intervals: $[0,1],[0,2]$, $\ldots[0, n], \ldots$. While associated with function $f$, each such a finite interval forms an m-history, since $\langle[0, n], f\rangle$ is consistent (because there is a real number above that interval). Yet obviously none of the finite intervals is maximal, whereas $\left\langle\Re^{+} f\right\rangle$ is maximal, but not consistent, and hence not an m-history.

A BST model exemplifying a similar feature has as the base set the real plane $\Re^{2}$, with Minkowskian ordering. ${ }^{3}$ This is a BST model with a single BST history and a BCont model with no choice events. Hence it per-

\footnotetext{
${ }^{3}$ That is, $\left\langle t_{1}, x_{1}\right\rangle \leqslant\left\langle t_{2}, x_{2}\right\rangle$ iff $\left(t_{2}-t_{1}\right)^{2} \geqslant\left(x_{2}-x_{1}\right)^{2}$ and $t_{1} \leqslant t_{2}$.
} 
mits only a single pointer function $f$. Consider now the chain of horizontal intervals: $\left[e_{0}, e_{1}\right],\left[e_{0}, e_{2}\right], \ldots\left[e_{0}, e_{n}\right], \ldots$, where $e_{0}=\langle 0,0\rangle, e_{1}=\langle 0,1\rangle, \ldots$ $e_{n}=\langle 0, n\rangle$, etc., and the first coordinate is temporal and the second-spatial. Each $\left\langle\left[e_{0}, e_{n}\right], f\right\rangle$ is an m-history, and each interval $\left[e_{0}, e_{n}\right]$, with its common future added, ${ }^{4}$ is an upward directed subset of $\Re^{2}$. Yet, $\left\langle\bigcup_{n \in N}\left[e_{0}, e_{n}\right], f\right\rangle$ is not an m-history, though $\bigcup_{n \in N}\left[e_{0}, e_{n}\right]$ with its common future added is an upward directed subset of $\Re^{2}$.

\subsection{Axioms}

We proceeded heedlessly without paying any attention to postulates of our BCont theory. Our policy is to refurbish any BST axiom that is violated in our construction. Here is a result of this policy, a tentative list of postulates.

\section{Definition 8 (model of BCont)}

A model of the theory of branching continuations (BCont) is a pair $\mathcal{W}=$ $\langle W, \leqslant\rangle$ that satisfies the following postulates:

1. $\langle W, \leqslant\rangle$ is a non-empty partially ordered set;

2. the ordering $\leqslant$ is dense on $W$;

3. $W$ has no maximal elements;

4. for every $x, y, e \in W$, if $e \nless x$ and $e \nless y$, then $x$ and $y$ are snake-linked in the subset $W_{\nexists e}:=\left\{e^{\prime} \in W \mid e^{\prime} \ngtr e\right\}$ of $W$ (though there might be many possible continuations, there can be at most one possible antinuation);

5. if $x, y \in W$ and $W_{\leqslant x y}:=\{e \in W \mid e \leqslant x \wedge \leqslant y\} \neq \emptyset$, then $W_{\leqslant x y}$ has a maximal element,

6. For every $x_{1}, x_{2} \in W$, if $\forall c: c \in C E \rightarrow c \nless x_{i}$, then $x_{1}$ and $x_{2}$ are snake-linked in the subset $W_{\ngtr C E}:=\{e \in W \mid \forall c \in C E e \ngtr c\}$ of $W$.

Postulates (1) - (3) are like in BST. Clause (4) requires that any two events that are not above some third event, are snake-linked in the region not-above $e$. This intends to enforce a condition analogous to downward closedness of BT/BST histories which says that if $e$ belongs to history $h$, then anything below $e$ belongs to $h$ as well. To see this, observe that we may define possible anti-nuations of events exactly as we defined possible continuations of events.

\footnotetext{
${ }^{4}$ This is the triangle defined by $0 \leqslant t \leqslant \frac{n}{2}$ and $\left|\frac{n}{2}-x\right| \leqslant\left|\frac{n}{2}-t\right|$.
} 
Clause (4) then guarantees that any event has at most one possible antinuation. ${ }^{5}$

Condition (5) is to exclude branching without a choice event; it produces maximal elements of common pasts, which in appropriate cases serve as choice events. Clause (6) is similar to the BT/BST postulate that all histories intersect.

Note further that continuations are stable as we have the following simple consequence of transitivity of $\approx_{e}$ :

\section{Fact 9}

For every $x, y, x^{\prime}, y^{\prime}, e \in W$, if $x \approx_{e} y, x \approx_{e} x^{\prime}$ and $y \approx_{e} y^{\prime}$, then $x^{\prime} \approx_{e} y^{\prime}$.

Thus, if $x$ and $y$ belong to the same continuation of $e$, any events above $e$ that are comparable to either $x$ or $y$, belong to this continuation.

The postulates above enforce a pattern of branching that is similar to the branching of BST 1992. In this theory, branching histories always require a choice point which is identified with a maximal element in the overlap of the histories in question. Also, if $c$ is a choice point of two BST histories, then the events on the future light cone of $c$ do not belong to the overlap of the two histories.

Our tactic is to consider two structures, similar to models of BST 1992, yet violating some of this theory's axioms as these structures incorporate a pattern of branching not permitted by BST 1992. Our aim is to show that they violate the postulates of BCont as well.

To begin, consider two copies of $\Re^{2}$, with a selected (cartesian) coordinate system $x, t$, and with the Minkowskian ordering - see Figure (2). Let the two planes be pasted in the overlap of the areas strictly below line $t=x$ and strictly below line $t=-x$. This means that the half-lines $t=x, t=-x$, with $t \geqslant 0$ are not in the pasted region. In particular, point $\langle 0,0\rangle$ is not in the pasted region. We thus obtain two separate triangular planes, each having "its" separate zero point at the vertex. To provide a handy name, I will call them (separate) possible futures, yet a question emerges: of what are these the futures? There are no choice events in this structure as, for every event $z$ in the overlap, there is an event strictly above $z$ in the overlap that can be used to snake-link above $z$ any two events belonging to the separate possible futures - see Figure (2). Accordingly, $z$ has one possible continuation and hence is not a choice event. Note, however, that any two events, each from

\footnotetext{
${ }^{5}$ The relation of being snake-linked in the subset $W_{\Varangle e}$ of $W$ is an equivalence relation on $W_{\nexists e}$. Possible anti-nuations of $e$ are then defined as elements of the partition of $W_{\nexists e}$ by the relation of being snake-linked not-above $e$.
} 


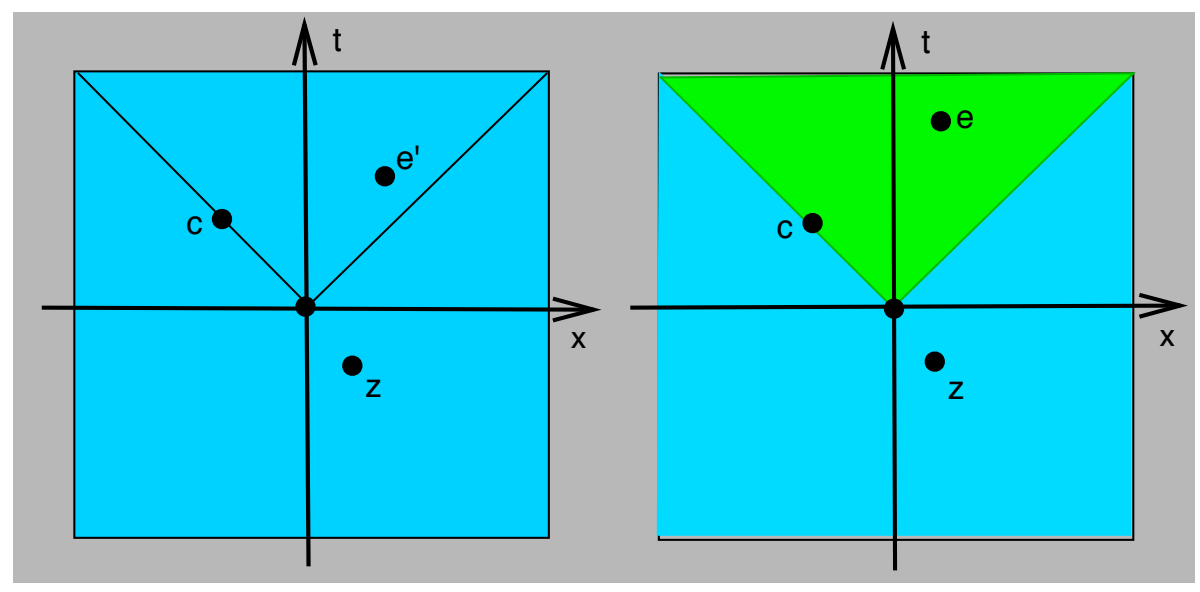

Figure 2: A diagram to represent three structures: (1) the vertex $\langle 0,0\rangle$ and events on its future light-cone do not belong to the overlap, (2) as in (1), with the exception of $c$ that belongs to the overlap, and (3) as in (1), with the exception of vertex $\langle 0,0\rangle$.

a different possible future, are not snake-linked within the union of these futures.

This structure violates axiom 5. Take two events, $e$ and $e^{\prime}$, each from a different possible future. The common past of $e$ and $e^{\prime}$, i.e., the set $W_{\leqslant x y}$, is non-empty. Yet, since the half-lines do not belong to the pasted region, there is no maximal element in the common past. This is a contradiction with axiom 5 .

To improve on the first construction, let us consider a seemingly better structure in which some single event, call it $c$, is located on one of the halflines above the vertex and belongs to the pasted region. Following an analogy with BST, it seems reasonable to require that no two events, each from a different possible future, be snake-linked within these futures. We cannot have this, however.

Pick arbitrary $y$ and $z$, each from a different possible future. Take then $s_{1}:=\sup (c, y)$ and $s_{2}:=\sup (c, z)$. Since we are in $\Re^{2}$ with Minkowskian ordering, the suprema exists. Clearly, $y<s_{1}, c<s_{1}, c<s_{2}, z<s_{2}$ and each of these events is within one of the possible futures. This is odd: the futures are separate, but they are linked by a snake-link. Yet, this construction contradicts axiom 5 , the argument being analogous to that given one paragraph above.

It remains to check on a structure like the initial one, yet in which point $\langle 0,0\rangle$ belongs to the overlap, but with no half-lines strictly above it. $\langle 0,0\rangle$ is a choice point as events from separate possible futures are not snake-linked 
above it; accordingly, these separate futures are the possible futures of $\langle 0,0\rangle$. This point is also a maximal element of the common past of any two events from different futures of $\langle 0,0\rangle$, i.e., it satisfies axiom 5 . Note finally that any attempt to add to the overlap a point on a half-line above $\langle 0,0\rangle$ will destroy $\langle 0,0\rangle$ as a choice event, as we argued one paragraph above.

The moral is that if one tries to implement branching on structures resulting from pasting Minkowski's space-times, the axioms of the present theory will enforce a pattern of branching assumed in BST 1992.

We finish this section with an argument that BCont postulates enforce an analogue of "no backward branching". The "no backward branching" condition has a clear sense in the presence of histories: for every events $x, y, z$, if $y<x$ and $z<x$, then there is a history $h$ to which both $y$ and $z$ belong. In BST 92 this immediately follows from the definition of history which identifies histories with maximal upward directed sets. Given that we have continuations and m-histories rather than histories, backward branching may mean two things:

1. for some $x, y, z$ such that $y<x$ and $z<x, y$ and $z$ belong to separate continuations of some event $e$;

2. some $y, z$, such that neither is above any choice event, are not snakelinked in region $W_{\nexists C E}:=\{e \in W \mid \forall c \in C E c \not e\}$.

Note that the second case is explicitly forbidden by axiom (6). But the first case cannot occur, either. For reductio, suppose $x, y, z$ are like in the premise, that is, for some $e: y \in H_{1}$ and $z \in H_{2}$, where $H_{1}, H_{2} \in \Pi_{e}$ and $H_{1} \neq H_{2}$. It follows that there is no snake-link above $e$ joining $y$ and $z$. But obviously there is a snake-link of this sort as $y<x, z<x$, and each $x, y, z$ is above $e$. Contradiction. Hence, there is no backward branching in the BCont theory.

To signal how the BCont theory can be further develop to analyze EPR phenomena and Bell's theorems, let us consider how to define the relations of compatibility and of being space-like related (SLR). As for compatibility, a first reflection is to say that two events are compatible if they do not belong to different continuations of any choice event. This will not do, however, unless we exclude the so-called modal funny business - cf. Belnap (2003). A typical case of this phenomenon is provided by a BST description of the EPR experiment, in which each of two incomparable choice events has two continuations, containing respectively results + and - , with only mixed joint results (i.e., +- and -+ ) being possible. Provided that in our model there are no other choice events, event $e$ immediately above the left choice event in its +- continuation and event $e^{\prime}$ immediately above the right choice event in its -+ continuation, do not belong to different continuations of any choice 
event, yet such events are paradigmatically incompatible. A remedy is to consider the set of choice events that are below $e$ or below $e^{\prime}$, which leads to the following definition:

\section{Definition 10 (compatibility, SLR)}

$e, e^{\prime} \in W$ are compatible iff they are snake-linked in the region lying above every choice event in the past of e or $e^{\prime}$. In symbols: $e, e^{\prime} \in W$ are compatible iff they are snake-linked in $R:=\left\{x \in W \mid \forall c\left(c \in C E \wedge\left(c<e \vee c<e^{\prime}\right)\right) \rightarrow\right.$ $c<x\}$.

$e, e^{\prime} \in W$ are SLR iff they are compatible but incomparable.

\subsection{Instants}

In BT Instants is a partition of the base set $W$. A linear ordering of Instants is derived there, by appealing to histories, from the partial ordering $\leqslant$ of base set $W$-cf. Belnap et al. (2001). In similar fashion, in some BST models we can derive spatiotemporal locations ( $a k a$ space-time points) - cf. Müller (2005). Following BT/BST, in the theory of BCont we define Instants to be a partition of $W$. Yet, as we have m-histories rather than histories, we cannot derive an ordering of Instants in this manner. Instead we postulate Instants to be linearly ordered by $\leqslant$. and require that ordering $\leqslant$ on $W$ and $\leqslant$. on Instants be properly related. We write $I(e)$ for that element of Instants to which $e$ belongs.

Postulate 11

$\forall e, e^{\prime} \in W: e \leqslant e^{\prime} \rightarrow I(e) \leqslant I\left(e^{\prime}\right)$.

In BT every element of Instants and every history intersect in a single event, but the analogous postulate on m-histories is unreasonable as m-histories can be as small as one likes. To consider a substitute, observe that the $\mathrm{BT}$ rationale for this requirement is that history is modally thin: no two incompatible events can belong to a history. Accordingly, two events from the same instant cannot belong to one history. Yet, m-histories are modally thin as well in the following precise sense: no two incompatible events can belong to set $A$, if $\langle A, f\rangle$ is an m-history for some pointer function $f$. We are thus lead to this postulate:

\section{Postulate 12}

For every m-history $\langle A, f\rangle$ and every $e_{1}, e_{2} \in A$ :

if $I\left(e_{1}\right) \leqslant \cdot I\left(e_{2}\right)$, then $e_{1} \leqslant e_{2}$. 
These two postulates entail that if $e_{1}, e_{2} \in A$ and $\langle A, f\rangle$ is an m-history for some $f \in \mathcal{P}$, then

$$
I\left(e_{1}\right) \leqslant \cdot I\left(e_{2}\right) \text { and } I\left(e_{2}\right) \leqslant \cdot I\left(e_{1}\right) \text { iff } I\left(e_{1}\right)=I\left(e_{2}\right) .
$$

It follows that $\leqslant \cdot$ restricted to set $A \subset W$ such that $\langle A, f\rangle$ is an m-history for some $f \in \mathcal{P}$, is a partial ordering. A further consequence is that no two elements of any such $A$ that $\langle A, f\rangle$ is an m-history, can belong to one and the same instant.

\section{Semantics without histories}

In this section we will test the theory, asking if it yields a reasonable semantics for a language with indexicals, tenses, and historical modalities, where reasonable means that it yields the same valid formulas as the BT semantics. We take BT for our reference theory, since it is simple and we have some intuitions concerning tenses. We do not have comparable intuitions concerning relativistic space-like relations, and for this reason an appeal to BST would be preposterous.

Now a BT theory supplemented by postulates - ordering is dense, there are no maximal elements, and the prior choice principle - satisfies the axioms of the last section. The BT theory so augmented has m-histories as well as histories (maximal chains). Yet, while using BT as a testing ground for our semantical theory, we will pretend it has no concept of history; we will thus recast $\mathrm{BT}$ semantical concepts accordingly. We further assume that the $\mathrm{BT}$ models here considered have Instants defined.

A novelty of Prior/Thomason BT semantics is that formulas are evaluated at event-history pairs where an event is assumed to belong to the history in question. Such pairs are written as $e / h$; accordingly $e / h \models \varphi$ is read as 'formula $\varphi$ is evaluated as true at evaluation point $e / h$ '. What should we use for evaluation points in the present theory? In particular, what should be used in place of history?

The obvious answer is: mini-histories but this calls for some caveats. Histories bring in a certain definiteness. Since they are global, the claim 'if $\varphi$ is true at $m / h$, then $\varphi$ is true at $m / h$ no matter how the course of events develops' sounds vacuously true. In our theory, a notion of how a course of events develops is rendered by a chain of m-histories, with later ones extending the earlier ones. Thus, to have a similar definiteness we might require that if $\varphi$ is true at $\langle\mathrm{e}, \mathrm{m}$-history $\rangle$, then it is true at any pair $\left\langle\mathrm{e}, \mathrm{m}\right.$-history $\left.{ }^{\prime}\right\rangle$, where m-history ${ }^{\prime}$ extends m-history. Note that for this requirement one needs first a notion of truth at a point of evaluation, and, in particular, the truth of 
future-tensed sentences. In fact, we assume a simpler requirement, namely that a point of evaluation is a pair $\langle e,\langle A, f\rangle\rangle$, where $e$ is an event, $\langle A, f\rangle$ is an m-history, and $e \leqslant \exists A$, where $e \leqslant \exists A$ abbreviates $\exists e^{\prime} \in A: e \leqslant e^{\prime}$. We will write such points of evaluations as $e / A f$. One might impose what seems a more natural requirement, i.e., $e \leqslant \forall A$, with $\leqslant_{\forall}$ explained analogously as above. But, while evaluating formulas with a future tense operator, we move to some event $e^{\prime}$ lying higher in the model. To produce an evaluation point $e^{\prime} / A^{\prime} f$, and in particular $A^{\prime}$, the alternative requirement forces us to toss out the past of $e$ from $A$. This we find too burdensome, and decide to sacrifice naturalness for simplicity. Accordingly, we assume this concept of evaluation points:

\section{Definition 13 (evaluation points)}

$\langle e,\langle A, f\rangle\rangle$ is an evaluation point, written as e/Af, iff (1) $e \in W$, (2) $\langle A, f\rangle$ is an m-history, and (3) $e \leqslant \exists A$.

What does it mean however that a sentence is true at such an evaluation point, as the latter involves a mini-history? We recommend an unabashedly ontological reading (in contrast to an epistemic interpretation which many would take as more natural). That is, we say: at event $e$, if mini-history $\langle A, f\rangle$ realizes, the sentence is true. Of course, we might be mistaken as to whether the mini-history in question will realize, or not. In a similar vein, we might be mistaken as to whether or not a sentence is true at $e$, if a given mini-history realizes. Still, as a mini-history is typically small and may have plenty of holes, we may have a premonition, a hunch, a forecast, or a scientific prediction that a given mini-history will realize.

This is strongly contrasted with our epistemic access to histories. Since histories are maximally large (and there are no holes in them, really), it is not in our power to form even an intuition of which history will realize. On a related issue, we believe that a context of use does not determine which mini-history will realize. There is no (future) mini-history of use, for quite similar reasons as there is no history of use in the BT semantics - cf. Belnap et al. (2001).

Since $\mathrm{m}$-histories can be small, it is preferable to work with metric tenses, like $F_{n}$ and $P_{m},{ }^{6}$ rather than open-ended tenses $F$ and $P$. (The latter can be re-introduced by quantifying over temporal periods.) For our testing purpose, consider then a mini language that has the familiar truth-conditional connectives: $\neg, \wedge, \vee$, and $\rightarrow$, metric tense operators $F_{n}$ and $P_{n}$, and modal operators Sett: and Poss:. With these operators, a single relevant aspect

\footnotetext{
${ }^{6}$ They are read as 'in $n$ units, it will be the case that ...' and ' $m$ units ago, it was the case that ...', respectively.
} 
of the context of use is the moment of use of a sentence, symbolized by $e_{C}$. Thus we take it that a formula is evaluated in a model, at the moment of its use, and at the point of evaluation. For a sentence considered as stand-alone, the $e$ of evaluation point $e / A f$ is identified with moment $e_{C}$ of use, whereas embedded sentences $e$ of $e / A f$ and $e_{C}$ may diverge. ${ }^{7}$

For structure $\mathfrak{S}$ we take a BT model with Instants; an interpretation function is $\mathfrak{J}:$ Atoms $\rightarrow \mathcal{P}(W)$ (where Atoms is the set of atomic formulas). ${ }^{8}$ The pair $\langle\mathfrak{S}, \mathfrak{J}\rangle$ is a model $\mathfrak{M}$. We will first define a technical notion of formula $\psi$ being fulfilled in model $\mathfrak{M}$ at the moment $e_{C}$ of use, and at point of evaluation $e / A f$. In symbols: $\mathfrak{M}, e_{C}, e / A f \approx \psi$. Later we will define the notion of a formula being definitely true.

We need, however, a few auxiliary notions for finally stating what it means that a formula is (definitely) true at an evaluation point. In order to warm up the reader for technical paragraphs to come, let us try to explain the underlying idea.

Whether a formula is (definitely) true at evaluation point $e / A f$, depends on how things stand appropriately high above $e$. The idea is that for a formula to be definitely true at $e / A f$, it should be fulfilled in every sufficiently long extension of $e / A f$. We thus need the notion of an extension of evaluation points and a semi-technical notion of fulfillment. We will define the following notions:

- An evaluation point $e / A f$ goes $n$-units-above $e$;

- One evaluation point is an $n$-units-above-e extension of some other evaluation point;

- A set of evaluation points is a fan (of evaluation points) defined by a given evaluation point;

- An evaluation point fulfills a formula;

- A formula is definitely true at an evaluation point.

Let us then get to work.

\footnotetext{
${ }^{7}$ For more on this issue, see Belnap et al. (2001) and Belnap (2007).

${ }^{8}$ Another option is to postulate that the interpretation function assigns a set of evaluation points to an atomic formula. Each option has its disadvantage. The situation exactly mimics a BT choice between an interpretation function sending atomic formulas into subsets of $W$, or into subsets of $W \times H i s t$, where Hist is the set of BT histories - cf. Thomason (1984).
} 
Definition 14 (extensions of an evaluation point)

$e / A^{\prime} f^{\prime}$ extends $e / A f$ iff $\langle A, f\rangle \preccurlyeq\left\langle A^{\prime}, f^{\prime}\right\rangle$.

$e / A f$ goes at least n-units-above $e(0 \leqslant n)$ iff $\exists e^{\prime} \in W: e^{\prime} \leqslant \exists A \wedge I\left(e^{\prime}\right)-$ $I(e)=n$;

$e / A^{\prime} f^{\prime}$ is an $n$-units-above-e extension of e/Af $(0 \leqslant n)$ iff $(1) e / A^{\prime} f^{\prime}$ extends $e / A f$ and (2) e/ $/ A^{\prime} f^{\prime}$ goes at least n-units-above $e$.

Observe that if an evaluation point is an $n$-units-above- $e$ extension of $e / A f$, it is also an $m$-units-above- $e$ extension of $e / A f$ for any $0<m \leqslant n$. The phrase "at least" implies that an $n$-units-above- $e$ extension of $e / A f$ can also be an $(n+m)$-units-above- $e$ extension of $e / A f$ for some $m>0$. Also an evaluation point can be an $n$-units-above- $e$ extension of itself. Note finally that starting with an arbitrary $e$ and an arbitrary m-history one might fail to produce an $n$-units-above- $e$ extension of an initial evaluation point.

With postulates (11) - (12) we are able to say what it means that mhistories are isomorphic instant-wise, and then define an auxiliary concept of a fan of evaluation points, which considerably simplifies the evaluation clause for the sentence with Sett: as a principal operator.

Definition 15 (fan of evaluation points)

m-histories $\left\langle A_{1}, f_{1}\right\rangle$ and $\left\langle A_{2}, f_{2}\right\rangle$ are isomorphic instant-wise iff $\forall e_{1} \in A_{1} \exists e_{2} \in$ $A_{2}: I\left(e_{1}\right)=I\left(e_{2}\right)$ and $\forall e_{2} \in A_{2} \exists e_{1} \in A_{1}: I\left(e_{1}\right)=I\left(e_{2}\right)$.

The fan $\mathcal{F}_{e, A f}$ of evaluation points produced by evaluation point $e / A f$ is a set of evaluation points:

$\mathcal{F}_{e, A f}:=\left\{e / A^{\prime} f^{\prime} \mid\langle A, f\rangle\right.$ and $\left\langle A^{\prime}, f^{\prime}\right\rangle$ are isomorphic instant-wise $\}$.

Note that while producing the fan out of a given evaluation point $e / A f$, we keep $e$ fixed and vary $A$ and $f$ subject to the condition of instant-wise isomorphism. And, since the elements of the fan are points of evaluation, $\left\langle A^{\prime}, f^{\prime}\right\rangle$ must be consistent and $e$ must be located below some element of $A^{\prime}$. Thus, many evaluation points permit only trivial fans, i.e., singletons of themselves.

\section{Definition 16 (point fulfills formula)}

1. if $\psi \in$ Atoms: $\mathfrak{M}, e_{C}, e / A f \approx \psi$ iff $e \in \mathcal{J}(\psi)$;

2. if $\psi$ is $\neg \varphi: \mathfrak{M}, e_{C}, e / A f \approx \psi$ iff it is not the case that $\mathfrak{M}, e_{C}, e / A f \approx \varphi$;

3. if $\psi$ is $\beta \vee \varphi: \mathfrak{M}, e_{C}, e / A f \approx \psi$ iff $\mathfrak{M}, e_{C}, e / A f \approx \beta$ or $\mathfrak{M}, e_{C}, e / A f \approx \varphi$;

4. and similarly for $\wedge$ and $\rightarrow$; 
5. if $\psi$ is $F_{n} \varphi: \mathfrak{M}, e_{C}, e / A f \approx \psi$ iff there is $e^{\prime} \in W$ such that $e^{\prime} \leqslant \exists A$ and $I\left(e^{\prime}\right)=I(e)+n$, and $\mathfrak{M}, e_{C}, e^{\prime} / A f \approx \varphi ;^{9}$

6. if $\psi$ is $P_{n} \varphi: \mathfrak{M}, e_{C}, e / A f \approx \psi$ iff there is $e^{\prime} \in W$ such that $e^{\prime} \leqslant \exists A$ and $I\left(e^{\prime}\right)=I(e)-n$, and $\mathfrak{M}, e_{C}, e^{\prime} / A f \approx \varphi ;$

7. if $\psi$ is Sett: $\varphi: \mathfrak{M}, e_{C}, e / A f \approx \psi$ iff for every evaluation point $e / A^{\prime} f^{\prime}$ from fan $\mathcal{F}_{e, A f}$ produced by e/Af: $\mathfrak{M}, e_{C}, e / A^{\prime} f^{\prime} \approx \varphi$.

The historical possibility operator (Poss:) is defined as usual as:

$$
\text { Poss }: \psi:=\neg \text { Sett }: \neg \psi \text {. }
$$

Finally, we have come to the definite truth: here is the notion of a formula being definitely true in a model, at a moment of use, and at an evaluation point, i.e., $\mathfrak{M}, e_{C}, e / A f \models \psi$.

\section{Definition 17 (definite truth)}

$\psi$ is definitely true at $\mathfrak{M}, e_{C}, e / A f$, in symbols $\mathfrak{M}, e_{C}, e / A f \models \psi$, iff there is an $n \geqslant 0$ such that for every $n$ units-above e extension $e / A^{\prime} f^{\prime}$ of $e / A f$ : $\mathfrak{M}, e_{C}, e / A^{\prime} f^{\prime} \approx \psi$;

$\psi$ is indefinite at $\mathfrak{M}, e_{C}, e / A f$, in symbols $\mathfrak{M}, e_{C}, e / A f ?=\psi$, iff there is no $n \geqslant 0$ such that for every $n$-units-above-e extension e/ $A^{\prime} f^{\prime}$ of e/Af: $\mathfrak{M}, e_{C}, e / A^{\prime} f^{\prime} \approx \psi$ or for every $n$-units-above-e extension $e / A^{\prime} f^{\prime}$ of e/Af: $\mathfrak{M}, e_{C}, e / A^{\prime} f^{\prime} \approx \neg \psi$.

As the first consequence of this definition, observe that for any formula $\psi$ and any evaluation point $e / A f$, exactly one of the following three options must hold:

$$
e / A f \models \psi \text { or } e / A f \models \neg \psi \text { or } e / A f ?=\psi \text {. }
$$

For, as for fulfillment of a formula at extensions of $e / A f$, these three cases exhaust all possibilities:

1. there is an $n \geqslant 0$ such that for every $n$ units-above $e$ extension $e / A^{\prime} f^{\prime}$ of $e / A f: e / A^{\prime} f^{\prime} \approx \psi$, or

2. there is an $n \geqslant 0$ such that for every $n$ units-above $e$ extension $e / A^{\prime} f^{\prime}$ of $e / A f: e / A^{\prime} f^{\prime} \not \nsim \psi$, or

3. there is no $n \geqslant 0$ satisfying (1) or (2) above.

\footnotetext{
${ }^{9}$ Note that the first condition after "such that" ensures that $e^{\prime} / A f$ is a legitimate point of evaluation.
} 
The first case means that $e / A f \models \psi$. Given the definition of fulfillment (clause on negation) and the first clause of definition (17), the second case is equivalent to $e / A f \models \neg \psi$. And given the definition of fulfillment (clause on negation) the third case is equivalent to $e / A f ?=\psi$.

We need to check on the stability of the notion of truth. We have the following lemma.

Lemma 18 If $\mathfrak{M}, e_{C}, e / A f \models \psi$, then for every extension $e / A^{*} f^{*}$ of evaluation point e/Af: $\mathfrak{M}, e_{C}, e / A^{*} f^{*}=\psi$.

Proof: It follows from the premise that there is an $n \geqslant 0$ such that for every $n$-units-above- $e$ extension $e / A^{\prime} f^{\prime}$ of $e / A f: \mathfrak{M}, e_{C}, e / A^{\prime} f^{\prime} \approx \psi$. Pick now an arbitrary extension $e / A^{\prime \prime} f$ " of $e / A^{*} f^{*}$. Let it be an $m$-units-above- $e$ extension of $e / A^{*} f^{*}$. There are now two cases: (i) $m<n$ and (ii) $n \leqslant m$.

Case (i). Consider all $(n-m)$-units-above-e extensions $e / A^{\prime \prime} f$ " of $e / A^{*} f^{*}$. By this construction, every $e / A$ " $f$ " is an $n$-units-above- $e$ extension of $e / A f$, so by the premise $\mathfrak{M}, e_{C}, e / A^{\prime \prime} f^{\prime \prime} \approx \psi$. Hence $\mathfrak{M}, e_{C}, e / A^{*} f^{*} \models \psi$.

Case (ii) For a given $k>0$, consider all $k$-units-above-e extensions $e / A$ " $f$ " of $e / A^{*} f^{*}$. Every $e / A^{\prime \prime} f$ " is an $(n+k)$-units-above- $e$ extensions $e / A f$, and hence an $n$-units-above- $e$. By the premise, for every $e / A$ " $f$ " of this sort, we have $\mathfrak{M}, e_{C}, e / A^{\prime \prime} f " \approx \psi$. Hence $\mathfrak{M}, e_{C}, e / A^{*} f^{*} \models \psi$.

Thus, a sentence definitely true at an evaluation point stays definitely true as we progress in time, whereas sentences indefinite at some evaluation points turn into definitely true or definitely false, whereby we call a sentence definitely false iff its negation is definitely true. In contrast, a sentence fulfilled at an evaluation point can cease to be fulfilled at an extension of this evaluation point, or vice versa. As for the dynamics of definite truth, the belief that every sentence sooner or later turns definitely true or definitely false is too optimistic, as the Instants may be exhausted.

Let us now state some simple observations on how definite truth, definite falsity and indefiniteness mesh together.

1. If $\psi$ is indefinite at a point, so is its negation.

2. if $\psi$ is indefinite at a point, $\psi \vee \varphi$ is either definitely true or indefinite at this point;

3. if $\psi$ is indefinite at a point, $\psi \wedge \varphi$ is either indefinite or definitely false at this point;

4. if $\psi$ is indefinite at a point, $\psi \rightarrow \varphi$ is either definitely true or indefinite at this point (same for $\varphi \rightarrow \psi$ ). 
5. settled cannot be indefinite: Sett: $\psi$ is definitely true or $\neg$ Sett : $\psi$ is definitely true.

A Pearcean future? No. In essence, for a sentence in the future tense, the Pearcean approach equates its being true at $e$ with this sentence being true at a later moment in every possible history to which $e$ belongs. A problem in this approach is that it fails to accommodate a distinction between what will happen and what will necessarily happen. In the present framework, we define definite truth by quantifying over possible extensions, which is similar to a Pearcean quantifying over possible future histories. One might thus suspect that we fail to draw a distinction which would indicate that our account is a version of the Pearcean approach. This is not correct, however: there is a difference between $F_{n} \phi$ and Sett : $F_{n} \phi$. To see this, consider $\mathrm{BT}+$ Instants model $\mathfrak{M}$ of Figure 3, with horizontal lines indi-

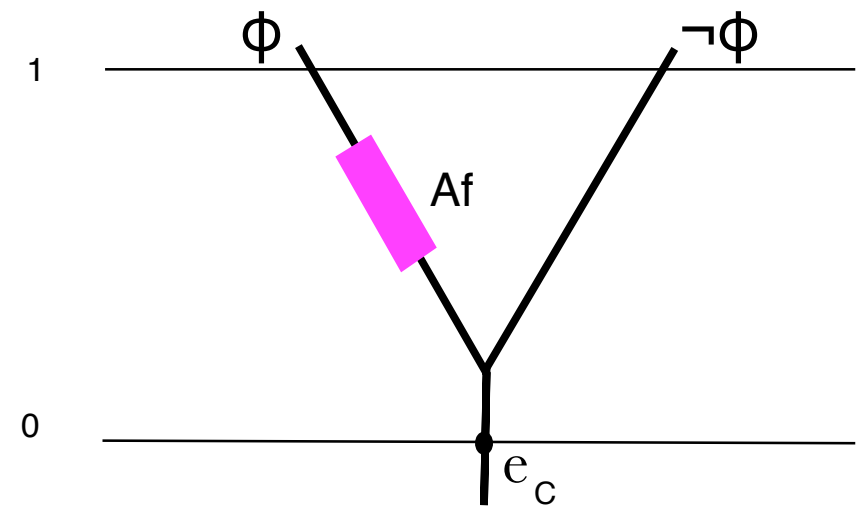

Figure 3: Difference between Sett: $F_{1} \phi$ and $F_{1} \phi$.

cating Instants, and the shadowed rectangle symbolizing the mini-history $\langle A, f\rangle . \quad F_{1} \phi$ is fulfilled by every 1 -units-above- $e_{C}$ extension of $e_{C} / A f$, and hence: $\mathfrak{M}, e_{C}, e_{C} / A f \models F_{1} \phi$. On the other hand, no matter how large $n \geqslant 0$ is, every fan produced by each $n$-units-above- $e_{C}$ extension of $e_{C} / A f$ has an 
element lying on the right branch of the model, where $\neg \phi$ is true at instant 1. Accordingly, we have: $\mathfrak{M}, e_{C}, e_{C} / A f \models \neg S e t t: F_{1} \phi$.

Is the past always settled? One premise of Diodor's Master Argument is that whatever is past or present, is settled, with a natural semantical reading: ( $\dagger$ ) from $P_{n} \varphi$, it follows that $S e t t: P_{n} \varphi$. What appears to be a convincing way of blocking Diodor's argument is von Kutschera's (1986) distinction between a sentence being about the past and the sentence being in the past tense. Diodor's premise is upheld if it concerns sentences about the past, but rejected if it concerns sentences in the past tense. Since a formula in the past tense is a formula with $P_{k}$ as the principal operator, we reject the implication $(\dagger)$. It can be easily seen that this rejection is also a consequence of the Prior/Thomason BT semantics. But is it a consequence of the present framework as well? Consider the model of Figure 4, with the symbol conventions explained as above. Let $\phi$ be an atomic formula and $e_{C}$ belong to Instant 1. $P_{1} F_{2} \phi$ is fulfilled by every 1 -units-above- $e_{C}$ extension of $e_{C} / A f$, and hence: $\mathfrak{M}, e_{C}, e_{C} / A f \models P_{1} F_{2} \phi$. On the other hand, for any $n \geqslant 0$, every fan produced by each $n$-units-above- $e_{C}$ extension of $e_{C} / A f$ has an element lying on the right branch of the model, where $\neg \phi$ is true at instant 2. Accordingly, we have: $\mathfrak{M}, e_{C}, e_{C} / A f \models \neg S e t t: P_{1} F_{2} \phi$. On the other hand, if $P_{1} \varphi$ is definitely true, so is $\operatorname{Sett}: P_{1} \varphi$, for $\varphi$ atomic. Thus, not every sentence in the past tense has a settled truth-value.

"Einstein was born a Nobel Prize winner" This conundrum sentence is ascribed to Arthur Prior. Let us suppose it is asserted now (in 2009) and that the assertor grants that Einstein might have failed to receive the Nobel Prize. Despite this indeterminism, the sentence appears to be settled true now. That is, it is settled (now) that it was true one hundred years ago that Einstein would receive the Nobel Prize (in 1921). In symbols, Sett : $P_{100} F_{12} \varphi$. This is compatible with the sentence that one hundred years ago it was not settled that Einstein would receive the Nobel Prize in 1921. We thus want to show that Sett: $P_{100} F_{12} \varphi$ does not imply $P_{100}$ Sett : $F_{12} \varphi$. Consider Figure (5). Every element of every fan produced by any extension of $e_{C} / A f$ lies on the left branch of the model, where $\phi$ holds. Hence, $\mathfrak{M}, e_{C}, e_{C} / A f \models S e t t: P_{100} F_{12} \phi$. On the other hand, no matter how large $n$ is, every extension $e_{C} / A^{\prime} f^{\prime}$ of $e_{C} / A f$ fails to fulfill $P_{100}$ Sett : $F_{12} \phi$. Namely, for every $e^{\prime}$ such that $e^{\prime}<e_{C}$ and $e^{\prime}$ belongs to Instant 1909, some element of the fan produced by $e^{\prime} / A^{\prime} f^{\prime}$ is on the right branch, where $\neg \phi$ holds. Accordingly, Sett : $F_{12} \phi$ is not fulfilled at $\mathfrak{M}, e_{C}, e^{\prime} / A^{\prime} f^{\prime}$, and 


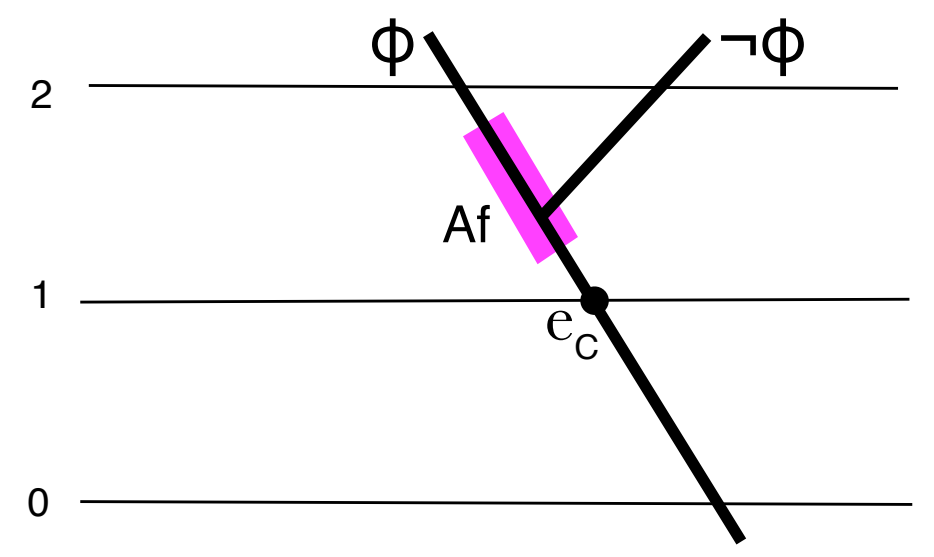

Figure 4: $\mathfrak{M}, e_{C}, e_{C} / A f \models P_{1} F_{2} \phi$ but $\mathfrak{M}, e_{C}, e_{C} / A f \models \neg S e t t: P_{1} F_{2} \phi$.

hence $P_{100}$ Sett : $F_{12} \phi$ is not fulfilled at $\mathfrak{M}, e_{C}, e_{C} / A^{\prime} f^{\prime}$. As $e_{C} / A^{\prime} f^{\prime}$ is an arbitrary $n$-units-above $e$ extension of $e_{C} / A f$ (for any arbitrarily large $n$ ), $\mathfrak{M}, e_{C}, e_{C} / A f \models \neg P_{100}$ Sett: $F_{12} \phi$.

Double time reference. The double time reference is a BT/BST tool, put forward by Belnap (2001) to explain how something that was not settled true at one event, becomes settled true at an appropriately later event. I phrase this intentionally vague since a part of the problem is what is that object which turns into settled truth?

In the BT framework, with metric rather than open tenses (to make it more similar to BCont theory), the following variation of Belnap's (2001) story illustrates this problem. (Note that in the next paragraph $\models$ stands for the BT truth at an event/history pair!)

At $e_{1}$ Themistocles makes the promise - "Tomorrow Themistocles will fight a sea battle" - in symbols: $F_{1} \varphi$. It is reasonable to assume that the core of the promise, " $F_{1} \varphi$ ", is not a settled truth at the event the promise was made, and also that the promise is not vitiated from the start. So:

$$
e_{1} / h \not=S e t t: F_{1} \varphi \text { and } e_{1} / h^{\prime} \models F_{1} \varphi \text { for some } h^{\prime} \in H i s t .
$$




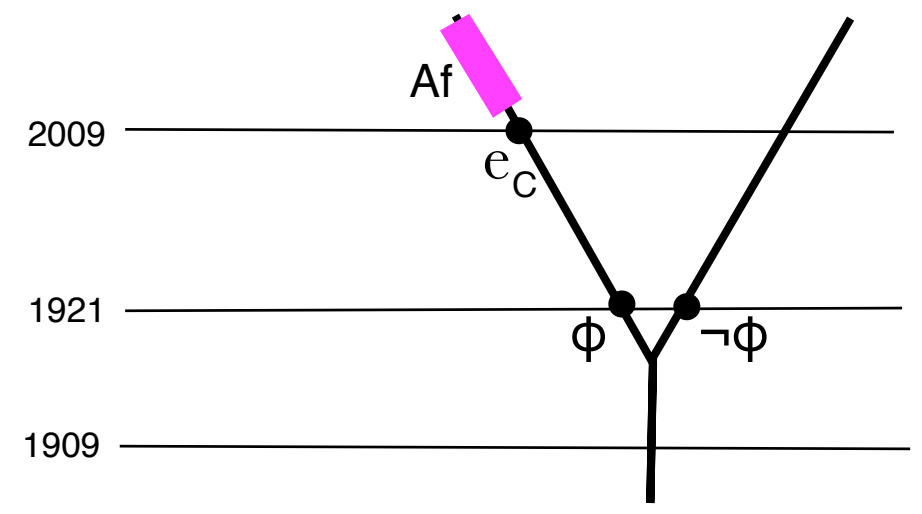

Figure 5: From Sett: $P_{100} F_{12} \phi$ it does not follow that $P_{100} S e t t: F_{12} \phi$.

The event at which the promise is satisfied is one $e_{2}$ at which it is settled true that Themistocles fights the battle, $e_{2} / h=S e t t: \varphi$. But what does it mean, generally speaking, that the promise made at $e_{1}$ is satisfied at $e_{2}$ ? The double-time reference yields this verdict:

The promise " $F_{1} \varphi$ " made by Themistocles at $e_{1}$ is satisfied at a later event $e_{2}$ iff $\forall h:\left(e_{2} \in h \rightarrow e_{1} / h \models F_{1} \varphi\right)$.

It follows that at event $e_{2}$ (at which the promise made at $e_{1}$ is satisfied) we have $e_{2} / h \models S e t t: P_{k} F_{1} \varphi$, where $k=I\left(e_{2}\right)-I\left(e_{1}\right)>0$.

We may introduce a similar device in the present framework. (Warning: from now on, we are again using $\models$ and $\approx$ of the BCont theory.)

The promise " $\psi$ " made by Themistocles at $e_{1}$ is satisfied at a later event $e_{2}$, i.e., $e_{1}<e_{2}$, iff

$$
\forall\langle A f\rangle:\langle A f\rangle \in m H i s t \wedge e_{2} \leqslant_{\exists} A \rightarrow e_{1} / A f \models \psi .
$$

We check if $(\dagger) e_{2} / A f \models S e t t: P_{k} \psi$, where $k=I\left(e_{2}\right)-I\left(e_{1}\right)$. It follows from (*) that for some $n \geqslant 0$ for every $n$-units-up extension $e_{1} / A^{\prime} f^{\prime}$ of $e_{1} / A f$ : $e_{1} / A^{\prime} f^{\prime} \approx \psi$ and (since $e_{2}<_{\exists} A$ ): $e_{2} \leqslant \exists A^{\prime}$. Let $\mathcal{B}:=\{\langle B, g\rangle \in$ mHist 
$\left.e_{2}{ }_{\exists} B\right\}$. It follows that for every $\langle B, g\rangle \in \mathcal{B}: e_{1} / B g \approx \psi$. Further, for every $\langle B, g\rangle \in \mathcal{B}: e_{2} / B g \approx P_{k} \psi$. Note also that every element of the fan produced by $e_{2} / B g$ is in $\mathcal{B}$. This entails $e_{2} / B g \approx P_{k}$ Sett: $\psi$. Since this holds for every extension $e_{2} / B^{\prime} g^{\prime}$ of $e_{2} / B g$, as it belongs to $\mathcal{B}$, we get $e_{2} / B g \models P_{k} S e t t: \psi$.

\section{Conclusions}

This paper puts forward a possible-worlds theory, of a branching variety, that works in terms of possible continuations and mini-histories rather than in terms of possible worlds or histories. The theory has a rigor comparable to, and similar explanatory virtues as Belnap's (1992) BST. The theory can also be used as a semantical theory for languages with indexicals, tenses, and historical modalities. The account allows that some sentences are undecided. It thus seems that we have here a philosophical paradise on the cheap: an ontological theory for indeterminism (in the sense of an open future) that is shy about possible histories. The open problem (and a large project) is to produce an ensemble of branching manifolds, i.e., a kind of generalization of an individual manifold of general relativity. 


\section{References}

Belnap, N. (1992). Branching space-time. Synthese, 92:385434. 'Postprint' archived at PhilSci Archive, http://philsciarchive.pitt.edu/archive/00001003.

Belnap, N. (2001). Double time references: Speech-act reports as modalities in an indeterminist setting. Advances in Modal Logic Vol. 3, pages 1-21. F. Wolter, H. Wansing, M. de Rijke, and M. Zakharyaschev (eds.).

Belnap, N. (2003). No-common-cause EPR-like funny business in branching space-times. Philosophical Studies, 114:199-221.

Belnap, N. (2007). An indeterminist view on the parameters of truth. In Müller, T., editor, Philosophie der Zeit, pages 87-113. Klostermann, Frankfurt a.M.

Belnap, N., Perloff, M., and Xu, M. (2001). Facing the Future. Oxford University Press, Oxford.

Kowalski, T. and Placek, T. (1999). Outcomes in branching space-time and GHZ-Bell theorems. British Journal for the Philosophy of Science, 50:349375 .

Müller, T. (2005). Probability theory and causation: a Branching SpaceTimes analysis. British Journal for the Philosophy of Science, 56(3):487520.

Placek, T. (2002). Indeterminism is more than enough. In Placek, T. and Butterfield, J., editors, Nonlocality and Modality, NATO Science Series, pages 317-342, Dordrecht. Kluwer Academic Publisher.

Thomason, R. H. (1984). Combinations of tense and modality. In Gabbay, D. and Guenthner, G., editors, Handbook of Philosophical Logic, vol. 2: Extensions of Classical Logic, pages 135-165. D. Reidel Publishing Company, Dordrecht.

von Kutschera, F. (1986). Zwei Modallogische Argumente für den Determinismus: Aristoteles und Diodor. Erkenntnis, 24:203-217. 